\title{
Power-Efficient Space Shift Keying Transmission via Semidefinite Programming
}

\author{
Adrian Garcia-Rodriguez, Christos Masouros, and Lajos Hanzo
}

\begin{abstract}
Space shift keying (SSK) transmission is a lowcomplexity complement to spatial modulation (SM) that solely relies on a spatial-constellation diagram for conveying information. The achievable performance of SSK is determined by the channel conditions, which in turn define the minimum Euclidean distance (MED) of the symbols in the received SSK constellation. In this contribution we concentrate on improving the power efficiency of SSK transmission via symbol pre-scaling. Specifically, we pose a pair of related optimization problems for a) enhancing the MED at reception while satisfying a given power constraint at the transmitter, and $b)$ reducing the transmission power required for achieving a given MED. The resultant optimization problems are NP-hard, hence they are subsequently reformulated and solved via semidefinite programming. The results presented demonstrate that the proposed pre-scaling strategies are capable of enhancing the attainable performance of conventional SSK, while simultaneously extending its applicability and reducing the complexity of the existing pre-scaling schemes.

Index Terms-Space shift keying, semidefinite programming, pre-scaling.
\end{abstract}

\section{INTRODUCTION}

Both space shift keying (SSK) and spatial modulation (SM) aim for reducing the hardware complexity of conventional spatial multiplexing [1], [2]. Specifically, both SSK and SM rely on encoding information into the active antenna indices, which allows reducing the number of radio frequency (RF) chains employed for transmission, when compared to the family of classic spatial multiplexing schemes [1], [2]. A theoretical characterization of the error rates experienced by SSK, where information is solely conveyed via a spatialconstellation diagram, and SM has been provided in [3], [4]. The development of detection algorithms for improving the attainable performance has also constituted the focus of intense research [5]-[7].

A parallel line of research has concentrated on the design of constellation shaping schemes for both SSK and SM [8]-[13]. In this context, [8] analyzes the design of amplitude and phase constellations for minimizing the average bit error probability of SM, whereas [9] analytically studies the achievable transmit diversity order under different design conditions. SSK's particular characteristic of solely carrying information in the spatial domain has also been exploited for the design of constellation shaping strategies in [10]-[13].

The maximization of the minimum Euclidean distance (MED) in the resultant SSK and SM constellations via symbol pre-scaling has been the focus of [14]-[16]. In particular, the pre-scaling strategies developed in [14], [15] rely on forcing the received SM constellation to resemble a classic quadrature amplitude modulation (QAM) constellation from an intersymbol distance perspective. However, the employment of the regimes in [14], [15] may severely affect the system's signal-to-noise ratio (SNR) due to the stringent requirement of inverting the channel coefficients, which may become critical for ill-conditioned channels. The scheme introduced in [16] mitigates this problem by solely applying a phase shift by the pre-scaling procedure. However, the above designs only consider a single antenna at the receiver, which in turn simplifies both the characterization and shaping of the received SM constellation.

The application of pre-scaling strategies to the more intricate multiple-input multiple-output (MIMO) systems has been promulgated in [17]-[20]. In particular, the schemes of [17], [18] propose opportunistic power allocation methods for both SSK and generalized SSK for the sake of improving their performance, which implies that only the amplitude of the transmit signals is modified. By contrast, simultaneous phase and amplitude pre-scaling is considered in the constellation randomization (CR) technique of [19]. This low-complexity scheme relies on generating $D$ complex-valued scaling factors off-line, and subsequently employing those specific scaling factors that maximize the MED. Moreover, the schemes introduced in [20], which have been developed in parallel to our work [21], further improve the performance by employing a successive convex approximation technique for solving the resultant optimization problems for maximizing the MED.

Against the above contributions, in this paper we consider the optimization of the pre-scaling factors for SSK transmission via semidefinite programming. Specifically, we recast the original NP-hard optimization problems for the sake of maximizing the performance of SSK transmissions via semidefinite relaxation (SDR). This guarantees the applicability of the proposed pre-scaling designs to multi-antenna aided receivers by carefully adapting the schemes introduced in [14]-[16]. Additionally, the results shown in this contribution demonstrate that the approach adopted improves the performance of the strategy developed in [19] by taking into account the channel conditions in the design of the pre-scaling vectors, while it reduces the signal processing complexity of the algorithms advocated in [20], where multiple convex optimization problems had to be solved before reaching convergence. We note however that this is achieved at the cost of a modest performance loss for the SSK systems considered.

\section{Space Shift Keying With Pre-Scaling}

The system model considered throughout this paper is comprised of a transmitter having $N_{t}$ antennas, and a receiver 


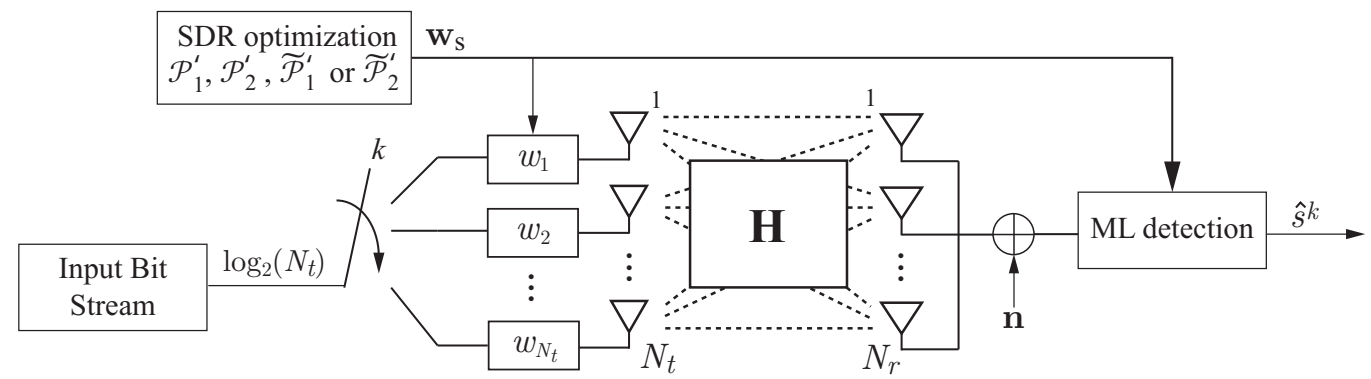

Fig. 1. Block diagram of the SSK communication scheme with pre-scaling and ML detection.

equipped with $N_{r}$ antennas, as shown in Fig. 1. The SSK transmitter activates a single antenna based on the input bit stream, hence conveying a total of $B=\left\lfloor\log _{2}\left(N_{t}\right)\right\rfloor$ bits per channel use, where $\lfloor\cdot\rfloor$ denotes the floor function. The received signal $\mathbf{y} \in \mathbb{C}^{N_{r} \times 1}$ can be expressed as

$$
\mathbf{y}=\sqrt{\rho} \mathbf{H W} \mathbf{e}_{k}+\mathbf{n}=\sqrt{\rho} \mathbf{h}_{k} w_{k}+\mathbf{n},
$$

where $\mathbf{e}_{k} \in \mathbb{C}^{N_{t} \times 1}$ is the $k$-th column of the identity matrix $\mathbf{I}_{N_{t}}$, and $\mathbf{n} \in \mathbb{C}^{N_{r} \times 1} \sim \mathcal{C N}\left(\mathbf{0}, \mathbf{I}_{N_{r}}\right)$ denotes the ubiquitous additive white-Gaussian noise vector. Moreover, $\mathbf{H} \in \mathbb{C}^{N_{r} \times N_{t}} \sim \mathcal{C N}\left(\mathbf{0}, \mathbf{I}_{N_{r}} \otimes \mathbf{I}_{N_{t}}\right)$ represents the Rayleigh communication channel considered and $\mathbf{W} \in \mathbb{C}^{N_{t} \times N_{t}}=$ $\operatorname{diag}(\mathbf{w})$ is a diagonal matrix, with its $k$-th diagonal coefficient given by $w_{k}$. In the previous expressions, $\otimes$ represents the Kronecker product and $\sim$ denotes "distributed as", while $\rho$ represents the average receive signal-to-noise ratio (SNR) per receive antenna, provided that $\mathbf{w}^{H} \mathbf{w}=N_{t}$. We note that the single-RF chain benefit of SSK transmission is preserved when pre-scaling is employed, and that the transmitted symbols solely rely on the instantaneous channel coefficients, but not on the alphabet and input bits.

The optimal detection strategy of the receiver obeys the maximum likelihood (ML) criterion of

$$
\hat{k}=\arg \min _{k}\left\|\mathbf{y}-\sqrt{\rho} \mathbf{h}_{k} \hat{w}_{k}\right\|^{2},
$$

where $\mathbf{h}_{k}$ is the $k$-th column of $\mathbf{H}$ and $\hat{w}_{k}$ denotes the $k$-th pre-scaling coefficient employed for reception. In the following we assume that the pre-scaling coefficients $\hat{w}_{k}$ and $w_{k}$ are computed independently at both the transmit and the receive sides based on the perfect channel estimates. Hence, no feedforwarding of the pre-scaling coefficients prior to data transmission is required.

The performance of the SSK transmission scheme considered is determined by the pairwise error probabilities [3]

$$
P\left(\mathbf{e}_{k} \rightarrow \mathbf{e}_{m} \mid \mathbf{H}\right)=Q\left(\sqrt{\frac{\rho}{2}\left\|\mathbf{h}_{k} w_{k}-\mathbf{h}_{m} w_{m}\right\|^{2}}\right), m \neq k,
$$

where $Q(\cdot)$ represents the $Q$-function, $Q(u)=$ $\frac{1}{\sqrt{2 \pi}} \int_{u}^{\infty} e^{-\frac{t^{2}}{2}} d t$, and $m, k \in 1, \ldots, N_{t}$, denote the specific index of the antenna activated for transmission. It can be seen from (3) that the detection performance of SSK is conditioned by the MED of the received constellation symbols [3], which is given by

$$
\operatorname{MED}=\min _{m, k}\left\|\mathbf{h}_{k} w_{k}-\mathbf{h}_{m} w_{m}\right\|^{2}, m \neq k .
$$

The efficient design of the SSK pre-scaling coefficients based on the above metric constitutes the focus of this contribution.

\section{A. MED Maximization}

An appealing technique of improving the attainable performance of conventional SSK transmission relies on maximizing the MED, while satisfying the maximum power constraint. In this particular case, the optimal pre-scaling vector $\mathbf{w}_{\text {opt }}$ can be obtained as a solution of the optimization problem

$$
\begin{gathered}
\mathcal{P}_{0}: \underset{\mathbf{w}}{\operatorname{maximize}} \min _{\substack{m, k \\
m \neq k}}\left(\left\|\mathbf{h}_{k} w_{k}-\mathbf{h}_{m} w_{m}\right\|^{2}\right) \\
\text { subject to }\|\mathbf{w}\|^{2} \leq\left(P_{t} N_{t}\right) .
\end{gathered}
$$

The constraint in (5) ensures having an average transmission power per channel use of $\mathbb{E}\left\{w_{k}^{H} w_{k}\right\}=P_{t}, \forall k \in 1, \ldots, N_{t}$. The more tractable epigraph problem form of (5) is given by

$$
\begin{gathered}
\mathcal{P}_{1}: \underset{\mathbf{w}, d}{\operatorname{maximize}} d \\
\text { subject to }\left\|\mathbf{h}_{k} w_{k}-\mathbf{h}_{m} w_{m}\right\|^{2} \geq d, \quad \forall m \neq k \\
\|\mathbf{w}\|^{2} \leq\left(P_{t} N_{t}\right) .
\end{gathered}
$$

Here, $d$ represents the MED. Note that the above optimization problem is not convex w.r.t. the optimization variable $\mathrm{w}$ due to the existence of non-convex quadratic constraints [22], [23].

\section{B. Power Minimization}

A problem of similar character to the above consists of procuring the pre-scaling factors that minimize the average transmission power, while satisfying a given MED threshold $d$. This optimization problem can be expressed as

$$
\mathcal{P}_{2}: \underset{\mathbf{w}}{\operatorname{minimize}}\|\mathbf{w}\|^{2}
$$

subject to $\left\|\mathbf{h}_{k} w_{k}-\mathbf{h}_{m} w_{m}\right\|^{2} \geq d, \quad \forall m \neq k$.

\section{Pre-Scaling Optimization For SSK Via SEMIDEFINITE PROGRAMMING}

The NP-hard nature of the nonconvex quadratically constrained quadratic problems $\mathcal{P}_{1}$ and $\mathcal{P}_{2}$ motivates the development of potentially suboptimal reformulations [22]-[24]. In 

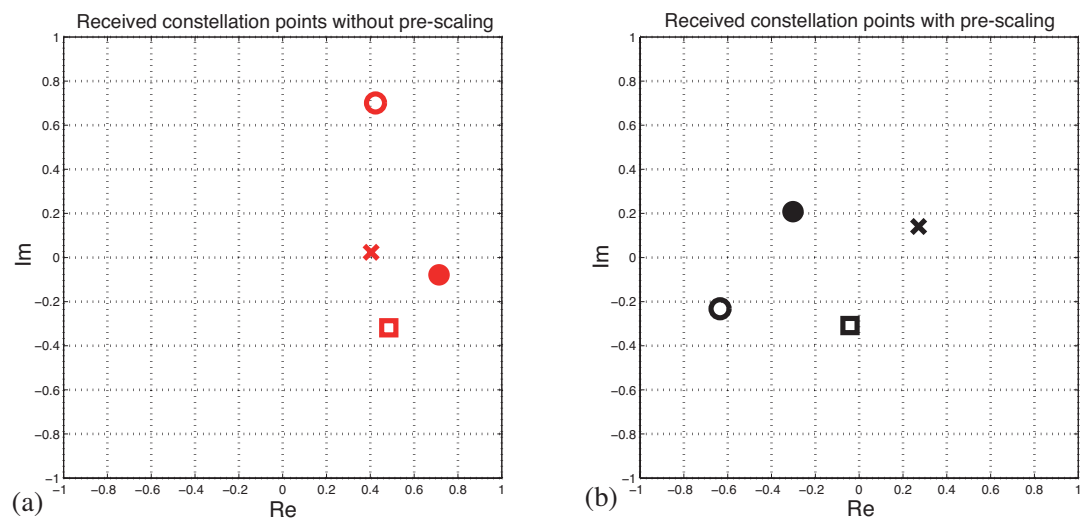

Fig. 2. Impact of pre-scaling in the received constellations for a $(4 \times 1)$-element MISO system. (a) Conventional SSK and (b) Pre-scaled SSK.

particular, we recast the optimization problems $\mathcal{P}_{1}$ and $\mathcal{P}_{2}$ as semidefinite programs by exploiting their resemblance to the sensor network location problem [22], [25], where the aim is to maximize the MED between adjacent sensor nodes.

\section{A. MED Maximization}

In the following we concentrate on reformulating the nonconvex optimization constraints of $\mathcal{P}_{1}$ via semidefinite relaxation [23]. Specifically, we first decompose the left-hand side of the quadratic constraints as

$$
\left\|\mathbf{h}_{k} w_{k}-\mathbf{h}_{m} w_{m}\right\|^{2}=\sum_{i=1}^{N_{r}}\left\|h_{(k, i)} w_{k}-h_{(m, i)} w_{m}\right\|^{2},
$$

where $h_{(k, i)}$ refers to the $i$-th entry of $\mathbf{h}_{k}$. The $i$-th term of the summation in (8) can be re-formulated as [25]

$$
\begin{aligned}
& \left\|h_{(k, i)} w_{k}-h_{(m, i)} w_{m}\right\|^{2}= \\
& \operatorname{Tr}\left(\mathbf{e}_{(k, m)}^{i}\left(\mathbf{e}_{(k, m)}^{i}\right)^{H} \mathbf{w w}^{H}\right)=\operatorname{Tr}\left(\mathbf{A} \mathbf{F}_{(k, m)}^{i}\right),
\end{aligned}
$$

where $\mathbf{A} \triangleq \mathbf{w w}^{H}, \mathbf{F}_{(k, m)}^{i} \triangleq \mathbf{e}_{(k, m)}^{i}\left(\mathbf{e}_{(k, m)}^{i}\right)^{H}$, and $\mathbf{e}_{(k, m)}^{i}$ is a vector with two non-zero entries in the positions specified by $k$ and $m$

$$
\mathbf{e}_{(k, m)}^{i}=\left[0, \ldots, h_{(k, i)}, \ldots,-h_{(m, i)}, \ldots, 0\right]^{T} .
$$

Let us define $\mathbf{F}_{(k, m)}$ as

$$
\mathbf{F}_{(k, m)} \triangleq \sum_{i=1}^{N_{r}} \mathbf{F}_{(k, m)}^{i} .
$$

Note that we have $\mathbf{F}_{(k, m)} \in \mathbb{H}^{N_{t}}, \forall k \neq m$, where $\mathbb{H}^{N_{t}}$ represents the set of $\left(N_{t} \times N_{t}\right)$-element complex-valued Hermitian matrices. Then, by substituting (8), (9) and (11) into (6), $\mathcal{P}_{1}$ can be recast as

$$
\begin{aligned}
\mathcal{P}_{1}: \underset{\mathbf{A}}{\operatorname{maximize}} & d \\
\text { subject to } & \operatorname{Tr}\left(\mathbf{F}_{(k, m)} \mathbf{A}\right) \geq d, k \neq m \\
& \operatorname{Tr}(\mathbf{A}) \leq\left(P_{t} N_{t}\right) \\
& \mathbf{A} \succeq 0, \operatorname{rank}(\mathbf{A})=1 .
\end{aligned}
$$

Here, $\mathbf{A} \succeq 0$ indicates that $\mathbf{A}$ is positive semidefinite, and we have considered that $\|\mathbf{w}\|^{2}=\operatorname{Tr}\left(\mathbf{w} \mathbf{w}^{H}\right)$. The above optimization problem is equivalent to that formulated in (6) and still remains NP-hard. However, a relaxed convex version of $\mathcal{P}_{1}$ can be obtained by dropping the non-convex constraint $\operatorname{rank}(\mathbf{A})=1$, which results in

$$
\begin{aligned}
\mathcal{P}_{1}^{\prime}: \underset{\mathbf{A}}{\operatorname{maximize}} d & \\
\text { subject to } & \operatorname{Tr}\left(\mathbf{F}_{(k, m)} \mathbf{A}\right) \geq d, \quad k \neq m \\
& \operatorname{Tr}(\mathbf{A}) \leq\left(P_{t} N_{t}\right), \quad \mathbf{A} \succeq 0 .
\end{aligned}
$$

The optimization problem (13) is convex in the optimization variables $\mathbf{A}$ and $d$, which facilitates the employment of efficient convex solvers. Although intrinsically suboptimal, in the sequel we show that the above semidefinite program is capable of remarkably enhancing the performance attained by conventional SSK transmission.

\section{B. Power Minimization}

Following a procedure akin to that employed for deriving $\mathcal{P}_{1}^{\prime}$ from $\mathcal{P}_{1}$, the semidefinite relaxation of $\mathcal{P}_{2}$ yields

$$
\begin{aligned}
\mathcal{P}_{2}^{\prime}: \underset{\mathbf{A}}{\operatorname{minimize}} & \operatorname{Tr}(\mathbf{A}) \\
\text { subject to } & \operatorname{Tr}\left(\mathbf{F}_{(k, m)} \mathbf{A}\right) \geq d, k \neq m, \\
& \mathbf{A} \succeq 0 .
\end{aligned}
$$

\section{Impact of Pre-Scaling on the Received Constellation}

Prior to characterizing the performance of the scheme considered, we illustrate the effect of solving the optimization problem $\mathcal{P}_{2}^{\prime}$ in the received constellation using an intuitive example. Specifically, Fig. 2(a) shows the received constellation, when conventional SSK transmission is employed, whereas Fig. 2(b) represents that under the same channel conditions, but applying the pre-scaling coefficients designed following $\mathcal{P}_{2}^{\prime}$ using $d=0.3$. In both figures, the distinct constellation symbols are illustrated by different geometrical shapes. The larger MED separation experienced by the constellation symbols of Fig. 2(b) demonstrates that the approach considered is indeed capable of enhancing the MED, hence improving the overall performance of conventional SSK. Moreover, it can be observed that the solution returned by the convex solver is 
TABLE I

EXPeCtation $(\mu)$ AND STANDARd DEVIATION $(\sigma)$ OF THE Figure OF MERIT $\mathcal{F}_{1}$ With $P_{t}=1$.

\begin{tabular}{|c||c|c||c|c||c|c||c|c|}
\hline$N_{t} / N_{r}$ & \multicolumn{2}{c||}{$S \boldsymbol{K} \boldsymbol{c}$} & \multicolumn{2}{c||}{ SSK-CR } & \multicolumn{2}{c|}{ SSK-SCA } & \multicolumn{2}{c|}{ SSK-SDR } \\
\hline \hline & $\mu$ & $\sigma$ & $\mu$ & $\sigma$ & $\mu$ & $\sigma$ & $\mu$ & $\sigma$ \\
\hline $2 / 1$ & 6.35 & 21.1 & 1.27 & 0.39 & 1 & 0 & 1 & 0 \\
\hline $2 / 2$ & 2.67 & 3.91 & 1.21 & 0.29 & 1 & 0 & 1 & 0 \\
\hline $4 / 2$ & 4.66 & 9.8 & 1.96 & 0.55 & 1.08 & 0.09 & 1.4 & 0.37 \\
\hline $4 / 3$ & 3.03 & 2.61 & 1.8 & 0.43 & 1.07 & 0.08 & 1.31 & 0.32 \\
\hline
\end{tabular}

capable of simultaneously reducing the average transmission power. However, the solutions obtained by solving the relaxed problems might become suboptimal as a consequence of removing the non-convex rank constraint. The characterization of this aspect in the pre-scaling scheme considered constitutes our focus in the following.

\section{Impact of the Problem Relaxation}

The pre-scaling vectors obtained by the SDR of the optimization problems $\mathcal{P}_{1}$ and $\mathcal{P}_{2}$ only coincide with those of $\mathcal{P}_{1}^{\prime}$ and $\mathcal{P}_{2}^{\prime}$ when we have $\operatorname{rank}(\mathbf{A})=1$ [22]. This implies that the pre-scaling vector $\mathbf{w}_{s}$ can be straightforwardly derived as

$$
\mathbf{w}_{s}=\mathbf{w}_{\text {opt }}=\mathbf{U} \boldsymbol{\Sigma}^{1 / 2},
$$

where $\mathbf{w}_{\text {opt }}$ denotes the optimal pre-scaling vector solution to $\mathcal{P}_{1}$ and $\mathcal{P}_{2}$, while $\mathbf{U}$ and $\boldsymbol{\Sigma}$ correspond to the eigenvectors and eigenvalues of $\mathbf{A}$ respectively, i.e., we have $\mathbf{A}=\mathbf{U} \boldsymbol{\Sigma} \mathbf{U}^{H}$. However, the above-mentioned ideal condition $\operatorname{rank}(\mathbf{A})=1$ is not always satisfied, and therefore we resort to randomization strategies for finding close-to-optimal solutions [22], [24]. Specifically, the pre-scaling vectors are obtained as [24]

$$
\mathbf{w}_{s}=c \mathbf{U} \mathbf{\Sigma}^{1 / 2} \mathbf{v}
$$

where $\mathbf{v}$ is a vector comprised of the exponential random variables characterized by, $v_{i}=e^{j \theta_{i}}, \theta_{i} \sim \mathcal{U}(0,2 \pi]$, which are uniformly distributed on the unit circle of the complex plane satisfying $\mathbb{E}\left\{\mathbf{v v}^{H}\right\}=\mathbf{I}_{N_{t}}$. Here, the constant $c$ guarantees that the problem constraints are satisfied. We remark that the solutions $\mathbf{w}_{s}$ obtained as a result of (15) and (16) are sub-optimal when we have $\operatorname{rank}(\mathbf{A}) \neq 1$, i.e., $\mathbf{W}_{s} \triangleq \mathbf{w}_{s} \mathbf{w}_{s}^{H} \neq \mathbf{w}_{\text {opt }} \mathbf{w}_{\text {opt }}^{H}$ [22], [24].

An accurate characterization of the impact of the above degradation should rely on contrasting the resultant value of the objective function, namely the MED attained or the optimal transmission power, obtained by the optimization problems $\mathcal{P}_{1}, \mathcal{P}_{2}$ and their relaxed versions $\mathcal{P}_{1}^{\prime}, \mathcal{P}_{2}^{\prime}$. This characterization is, however, impractical due to the computational hardness of deriving the optimal solution to the original problems $\mathcal{P}_{1}$ and $\mathcal{P}_{2}$ [22], [24]. For this reason, we characterize the impact of relaxation by exploiting that the value, $f^{\prime \star}$, of the objective function delivered by SDR provides a useful bound to the optimal problem [24]. Therefore, a relevant figure of merit $\mathcal{F}$ can be defined as [24]

$$
\mathcal{F}_{\{1,2\}}=\frac{f_{\{1,2\}}^{\prime \star}}{f_{\{1,2\}}^{s}},
$$

where $f_{\{1,2\}}^{\prime \star}$ denotes the specific value of the objective function in $\mathcal{P}_{\{1,2\}}^{\prime}$, when the solution directly retrieved by the solver $\mathbf{A}$ is employed, while $f_{\{1,2\}}^{s}$ corresponds to the particular value of the objective function obtained after applying (15) or (16), i.e. by employing $\mathbf{W}_{s}=\mathbf{w}_{s} \mathbf{w}_{s}^{H}$. In the above expressions, the subscripts refer to the optimization problem considered, i.e., $\mathcal{P}_{1}$ or $\mathcal{P}_{2}$. At this point we stress that the solutions retrieved by the solver $\mathbf{A}$ are different from those obtained after randomization $\mathbf{W}_{s}$ when we have $\operatorname{rank}(\mathbf{A}) \neq 1$. The figure of merit $\mathcal{F}$ in (17) can also be generalized both to SSK and to CR-aided SSK (SSK-CR) [19], as well as to SSK based on sucessive convex approximation (SSK-SCA) [21] to determine the solution's proximity to the optimal one.

Table I characterizes both the expectation and the standard deviation of $\mathcal{F}_{1}$, explicitly quantifying the degradation of the solutions provided by the schemes considered in this paper. In this particular case, $f_{1}^{\prime \star}$ and $f_{1}^{s}$ correspond to the MED obtained by employing $\mathbf{A}$ and $\mathbf{W}_{s}$ respectively. Note that $\mathcal{F}_{1} \geq 1$, since the MED bound $f_{1}^{\prime}$ obtained by the solver is always larger than or equal to the MED $f_{1}^{s}$ attained after randomization. $D=20$ candidate scaling vectors are considered for SSK-CR [19], [21]. Remarkably, the results of Table I show that both the proposed semidefinite relaxation (SSK-SDR) and SSK-SCA always achieve the optimal solution for $N_{t}=2$, which can be explained by the Shapiro-Barvinok-Pataki bound [20]. It can also be observed that the proposed SSK-SDR prescaling is capable of reducing both the expectation and the standard deviation of the figure of merit $\mathcal{F}$, when compared to conventional SSK and SSK-CR. In other words, the solutions retrieved by the proposed SSK-SDR are closer to the solution of the optimal problem $\mathcal{P}_{1}$. Indeed, the results of Table I indicate that the benefits offered by the proposed SDRbased technique become more pronounced for reduced system dimensions. Simultaneously, it can be seen that the SSK-SCA algorithm, which was published throughout the development of this work [21], is capable of providing better solutions than the proposed SSK-SDR scheme. Nonetheless, in the following we will show that the closer proximity of the SSK-SCA solutions to the optimal ones is achieved at the expense of a substantial increase in their computational complexity, and that the performance differences remain modest.

\section{NuMERICAL RESULTS}

Both the attainable performance and power efficiency improvements offered by the proposed strategy are analyzed in 


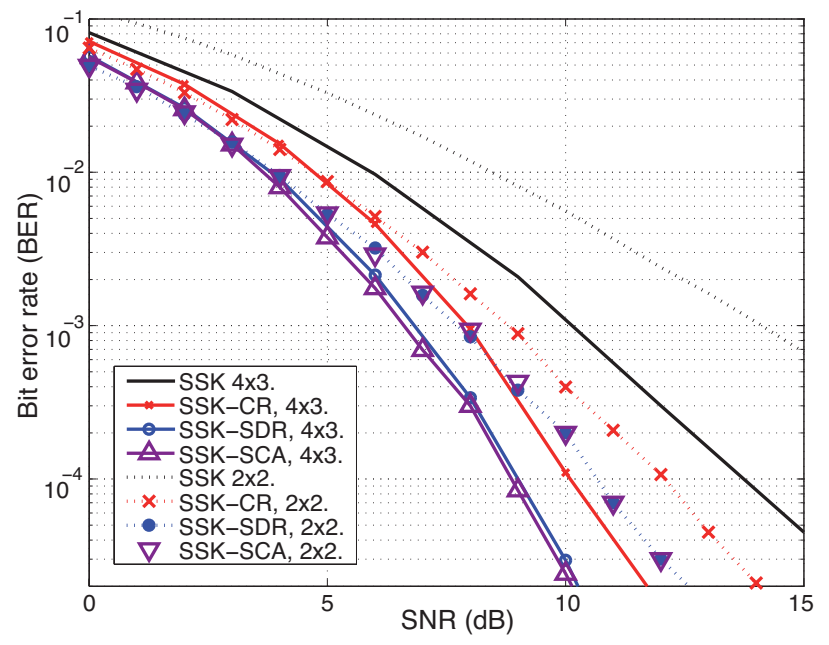

Fig. 3. BER vs. SNR for both a) $(2 \times 2)$ and b) $(4 \times 3)$ MIMO systems.

this section. Specifically, we compare the proposed scheme to conventional SSK transmission operating without prescaling (SSK), to the SSK constellation randomization scheme developed in [19] (SSK-CR), and to the sucessive convex approximation strategies of [20] (SSK-SCA). The number of random pre-scaling coefficients is $D=20$ for SSK-CR, as in Sec. III-D [19], [21]. Moreover, we consider the SSK-SCA algorithms to be converged when the Euclidean norm of the relative error for consecutive solutions satisfies $\xi \leq 10^{-3}$, which indicates a faster convergence than the $\xi \leq 10^{-5}$ value considered in [20]. This faster convergence is achieved without perceptible performance differences.

Fig. 3 shows the evolution of the bit error rates (BERs) upon increasing the SNR $(\rho$ in (1)) for both $(2 \times 2)$ - and $(4 \times 3)$-element MIMO systems. The results demonstrate that our pre-scaling strategies are able to substantially improve the performance attained by conventional SSK. Specifically, it can be seen that SSK-SDR reduces the error rates of both SSK and SSK-CR, while it slightly degrades the performance of the more complex SSK-SCA for the $(4 \times 3)$ MIMO system. We note that both SSK-SDR and SSK-SCA improve the performance w.r.t. SSK-CR, since they are capable of approaching the globally optimal solution for MIMO systems associated with $N_{t}=2$, as detailed in Sec. III-D.

The empirical probability density function (PDF) of the relative computational time required for obtaining the results of Fig. 3 for the $(4 \times 3)$ MIMO system is shown in Fig. 4 . We show the percentage of the relative times with respect to the maximum time required for the SSK-SCA algorithm for a fair comparison, since the absolute time measure depends on the specific computational capabilities. For this reason, the analysis of the computational time required has been tested using the same computational capabilities and without the influence of any other active processes. The results of Fig. 4 show that the proposed SSK-SDR pre-scaling technique offers a substantial complexity advantage over the SSK-SCA scheme. This can be explained by the SSK-SCA requirement of solving multiple convex problems before approaching convergence.
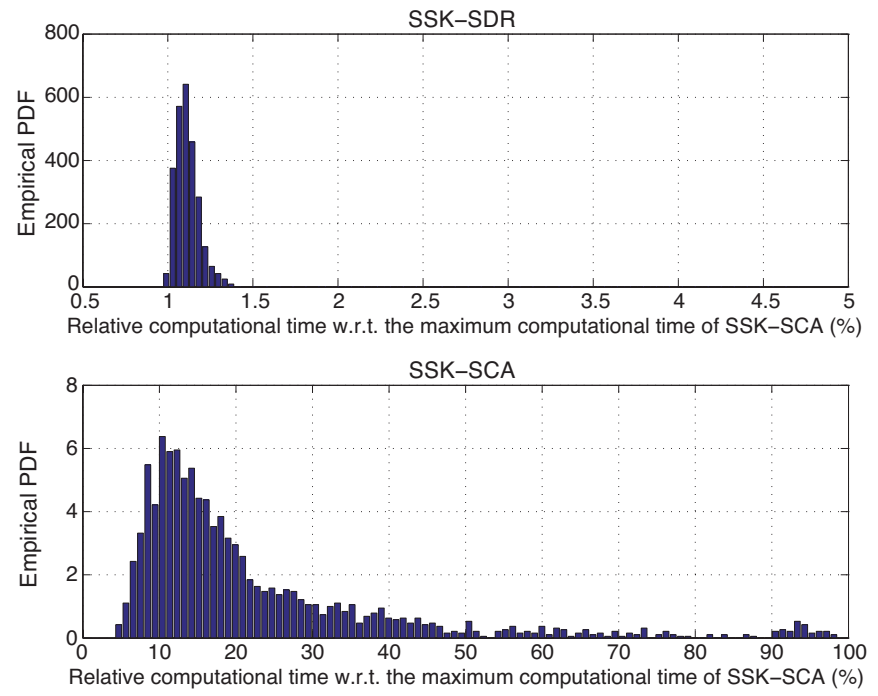

Fig. 4. Empirical PDF of the computational time required in (a) SSK-SDR and (b) SSK-SCA. $(4 \times 3)$ MIMO system.

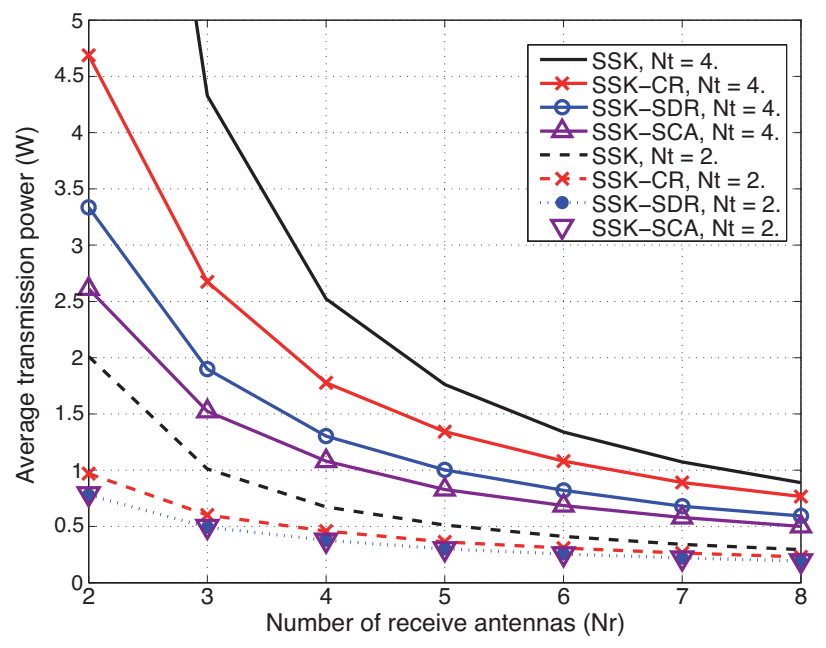

Fig. 5. Average transmission power vs number of receive antennas $N_{r}$ for systems with $N_{t}=\{2,4\}$ transmit antennas and MED threshold $d=2$.

We note that the computational time improvements manifest themselves both in the average time and its maximum, which is a critical parameter for real-time applications. Clearly, both the SSK-SDR and the SSK-SCA schemes are more complex than SSK-CR, since the computation of the pre-scaling factors is performed off-line for the latter. Nonetheless, SSK-SDR is capable of offering a compelling complexity-performance trade-off due to the performance improvements provided.

The results of Fig. 5 show the average transmission power of the systems considered upon increasing the number of receive antennas $N_{r}, d=2$, and $N_{t}=\{2,4\}$. Without loss of generality, we assume having an instantaneous maximum transmission power of 20 Watts, which may only be necessary for ill-conditioned channels and it is in line with the need of imposing a practical constraint on realistic power amplifiers. Fig. 5 clearly shows the advantages of incorporating a larger number of receive antennas for reducing the transmission power required to attain a given target performance. Moreover, 


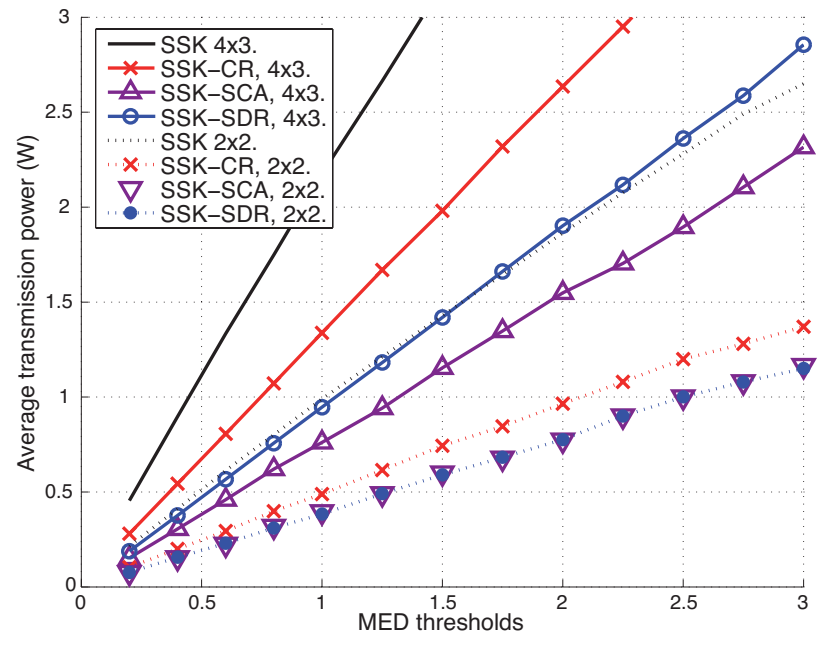

Fig. 6. Average transmission power vs MED thresholds $d$ for both a) $(2 \times 2)$ and b) $(4 \times 3)$ MIMO systems.

it can be seen from Fig. 5 that the differences in the transmitted power among the different pre-scaling strategies are maximized, when the number of receive antennas is reduced. For instance, Fig. 5 shows that the employment of SSK-SDR in a $(4 \times 4)$ MIMO system allows us to reduce the average transmission power by 0.5 Watts compared to SSK-CR, whereas SSK-SCA further improves the former by 0.25 Watts.

A more detailed view of the impact of varying the MED thresholds on the transmission power required can be observed in Fig. 6. This figure depicts the increase in the transmission power required for satisfying higher MED thresholds. The results of Fig. 6 show that the benefits of applying pre-scaling are maximized at high SNRs. It can also be observed that the proposed SSK-SDR is capable of outperforming SSK-CR, and that SSK-SCA is capable of further approaching the optimal pre-scaling in the scenarios considered, albeit at the expense of a higher computational complexity.

\section{Conclusions}

The efficient design of pre-scaling coefficients for enhancing the power efficiency of SSK transmission has been the focus of this work. The proposed approach is based on relaxing the optimal but NP-hard pre-scaling problems for obtaining a convex formulation that facilitates the application of standard convex solvers. The results derived have shown that the reformulated optimization problems are capable of enhancing both the BER performance and the energy efficiency of existing SSK schemes at a moderate complexity.

\section{ACKNOWLEDGMENT}

This work was supported by the Royal Academy of Engineering, UK and the EPSRC under grant EP/M014150/1.

\section{REFERENCES}

[1] P. Yang, M. Di Renzo, Y. Xiao, S. Li, and L. Hanzo, "Design guidelines for spatial modulation,” IEEE Commun. Surveys Tutorials, vol. 17, no. 1, pp. 6-26, Firstquarter 2015.

[2] P. Yang et al., "Single-carrier spatial modulation: A promising design for large-scale broadband antenna systems," IEEE Commun. Surveys and Tutorials, submitted.
[3] J. Jeganathan, A. Ghrayeb, and L. Szczecinski, "Spatial modulation: optimal detection and performance analysis," IEEE Commun. Letters, vol. 12 , no. 8, pp. 545-547, Aug 2008.

[4] M. Di Renzo and H. Haas, "Bit error probability of SM-MIMO over generalized fading channels," IEEE Trans. on Veh. Tech., vol. 61, no. 3, pp. 1124-1144, March 2012.

[5] A. Younis, S. Sinanovic, M. Di Renzo, R. Mesleh, and H. Haas, "Generalised sphere decoding for spatial modulation," IEEE Trans. on Commun., vol. 61, no. 7, pp. 2805-2815, July 2013.

[6] C. Masouros and L. Hanzo, "Dual layered MIMO transmission for increased bandwidth efficiency," IEEE Trans. on Veh. Tech., Accepted for publication, 2015.

[7] A. Garcia-Rodriguez and C. Masouros, "Low-complexity compressive sensing detection for spatial modulation in large-scale multiple access channels," IEEE Trans. on Commun., vol. 63, no. 7, pp. 2565-2579, July 2015

[8] P. Yang, Y. Xiao, B. Zhang, S. Li, M. El-Hajjar, and L. Hanzo, "StarQAM signaling constellations for spatial modulation," IEEE Trans. on Veh. Tech., vol. 63, no. 8, pp. 3741-3749, Oct 2014.

[9] M. Di Renzo and H. Haas, "On transmit diversity for spatial modulation MIMO: Impact of spatial constellation diagram and shaping filters at the transmitter,' IEEE Trans. on Veh. Tech., vol. 62, no. 6, pp. 2507-2531, July 2013.

[10] S. Sugiura, C. Xu, S. X. Ng, and L. Hanzo, "Reduced-complexity coherent versus non-coherent QAM-aided space-time shift keying," IEEE Trans. on Commun., vol. 59, no. 11, pp. 3090-3101, Nov. 2011.

[11] S. Sugiura and L. Hanzo, "On the joint optimization of dispersion matrices and constellations for near-capacity irregular precoded spacetime shift keying," IEEE Trans. on Wireless Commun., vol. 12, no. 1, pp. 380-387, January 2013.

[12] M. Maleki, H. Bahrami, S. Beygi, M. Kafashan, and N. Tran, "Space modulation with CSI: Constellation design and performance evaluation," IEEE Trans. on Veh. Tech., vol. 62, no. 4, pp. 1623-1634, May 2013.

[13] K. Ntontin, M. Renzo, A. Perez-Neira, and C. Verikoukis, "Adaptive generalized space shift keying," EURASIP Journal on Wireless Commun. and Networking, vol. 2013, no. 1, p. 43, 2013.

[14] J. Luna-Rivera, D. U. Campos-Delgado, and M. Gonzalez-Perez, "Constellation design for spatial modulation," Procedia Tech., vol. 7, pp. 71$78,2013$.

[15] X. Guan, Y. Cai, and W. Yang, "On the mutual information and precoding for spatial modulation with finite alphabet," IEEE Wireless Commun. Letters, vol. 2, no. 4, pp. 383-386, August 2013.

[16] C. Masouros, "Improving the diversity of spatial modulation in MISO channels by phase alignment," IEEE Commun. Letters, vol. 18, no. 5, pp. 729-732, May 2014.

[17] C.-H. Wu, W.-H. Chung, and H.-W. Liang, "Improved generalized spaceshift keying via power allocation," IEEE Commun. Letters, vol. 18, no. 7, pp. 1143-1146, July 2014.

[18] M. Di Renzo and H. Haas, "Improving the performance of space shift keying (SSK) modulation via opportunistic power allocation," IEEE Commun. Letters, vol. 14, no. 6, pp. 500-502, June 2010.

[19] C. Masouros and L. Hanzo, "Constellation-randomization achieves transmit diversity for single-RF spatial modulation," IEEE Trans. on Veh. Tech., under review, [Online]. Available: http://greeninterference.weebly.com/uploads/2/4/6/5/24652439/masouros_sm-constrand5.pdf.

[20] M.-C. Lee, W.-H. Chung, and T.-S. Lee, "Generalized precoder design formulation and iterative algorithm for spatial modulation in MIMO systems with CSIT," IEEE Trans. on Commun., vol. 63, no. 4, pp. 12301244, April 2015.

[21] A. Garcia-Rodriguez, C. Masouros, and L. Hanzo, "Pre-scaling optimization for space shift keying based on semidefinite relaxation," IEEE Trans. on Commun., Accepted for publication, 2015.

[22] Z.-Q. Luo, W.-K. Ma, A.-C. So, Y. Ye, and S. Zhang, "Semidefinite relaxation of quadratic optimization problems," IEEE Signal Processing Magazine, vol. 27, no. 3, pp. 20-34, May 2010.

[23] S. Boyd and L. Vandenberghe, Convex optimization. Cambridge university press, 2004.

[24] N. Sidiropoulos, T. Davidson, and Z.-Q. Luo, "Transmit beamforming for physical-layer multicasting," IEEE Trans. on Signal Processing, vol. 54, no. 6, pp. 2239-2251, June 2006.

[25] P. Biswas and Y. Ye, "Semidefinite programming for ad hoc wireless sensor network localization," in Third Int. Symposium on Information Processing in Sensor Networks (IPSN), April 2004, pp. 46-54. 